\title{
4 RULES AND PATTERNS FOR SECURITY IN WORKFLOW SYSTEMS
}

\author{
Silvana Castano and Maria Grazia Fugini
}

\begin{abstract}
Assignment of tasks to agents in a Workflow (WF) system should occur according to security policies regarding user authorizations to access data and documents through the WF tasks. This paper presents an approach to discretionary secure assignment of tasks to agents taking into account authorization constraints, in the framework of the WIDE (Workflow Interactive Development Environment) WF management system. The approach is based on the concepts of role, agent, and task, and on authorization patterns and rules. Security rules (or triggers) specify which actions (e.g., security warnings, logs, audit actions) should be taken when a security violation (event) occurs, following the ECA paradigm of active databases. A basic set of rules is provided in the abstracted form of authorization patterns which are generic rule skeletons to be properly instantiated to enforce authorization constraints in a given WF application.
\end{abstract}

\subsection{INTRODUCTION}

WFs are complex activities (or business processes) that involve the coordinated execution of several tasks to reach a common objective [8]. The design of WF applications requires the capability to cope also with security requirements taking into account the organization of users work and the structure of business processes [3]. Issues related to security in WFs, and distributed systems in general, have been receiving much interest in recent literature. A model to flexibly specify role-based authorization constraints in WF systems is described in [2]. Security in WFs is tackled in [9] where a discussion is provided regarding security controls in collaborative WFs (e.g., discretionary and 
mandatory access controls, and object-oriented security). In [5], automatic construction of authorizations in a federated database is described, supporting flexible cooperation and data sharing. Task-based authorization in distributed systems is discussed in [11] as a flexible and adaptable access control paradigm.

Our approach to WF security consists in specifying rules for the control of agent assignment to WF tasks in order to enforce authorization constraints on WF execution. Rules (or triggers) are composed of events, conditions, and actions (ECA paradigm [13]). The event part of the rule specifies violations to the considered authorization constraint, the condition part determines if the occurred event actually corresponds to a violation situation to be managed, while the action part specifies the reaction of the system to the occurred violation within the normal flow. The approach has been developed in the framework of WIDE (Workflow on Intelligent Distributed database Environment), an EEC Esprit Project aimed at realizing a WF management system on top of an active database, using rules as the exception modeling paradigm. In fact, WF design in WIDE consists in modeling the "normal behavior" of a WF as well as the exceptions arising as "predictable deviations of the normal behavior" of the WF itself.

Enforcing authorization constraints by means of rules in WFs can be complex, because the normal behavior and the anomalous situations have to be identified, together with their corresponding corrective actions. Following recent proposals in the software engineering area [7], in WIDE pre-defined authorization patterns are introduced to reduce the design effort related to WF security exception modeling and handling. Authorization patterns are rule skeletons modeling typical authorization constraint exceptions for WFs in given domains. Rule skeletons constituting a given pattern can be reused and adapted to a new WF application by instantiating them into triggers to be executed on the WIDE active database.

The main contribution of our work is related to the use of a trigger-based mechanism for authorization constraint enforcement and of a pattern-based formalism and associated catalog for trigger design. In the literature on WF security, authorization constraints for WFs have been recently studied (see, for instance, [2]) from a specification point of view, by providing a logic-based language for constraint specification that facilitates also system analysis. In this paper, the focus is more on the implementation of WF authorization constraints based on the active rule paradigm. Moreover, we provide authorization patterns in a catalog as a means to reuse the knowledge on authorization constraints when designing a new WF, to avoid the definition of triggers from scratch each time an authorization constraint must be enforced in a WF.

The paper is organized as follows. First, the WIDE model and the use of rules to WF specification are presented. Then, issues related to authorization constraints and triggers in WFs are discussed. A basic set of authorization patterns is described to enforce the most frequent authorization constraints regarding secure task execution. Issues related to constraint enforcement based 


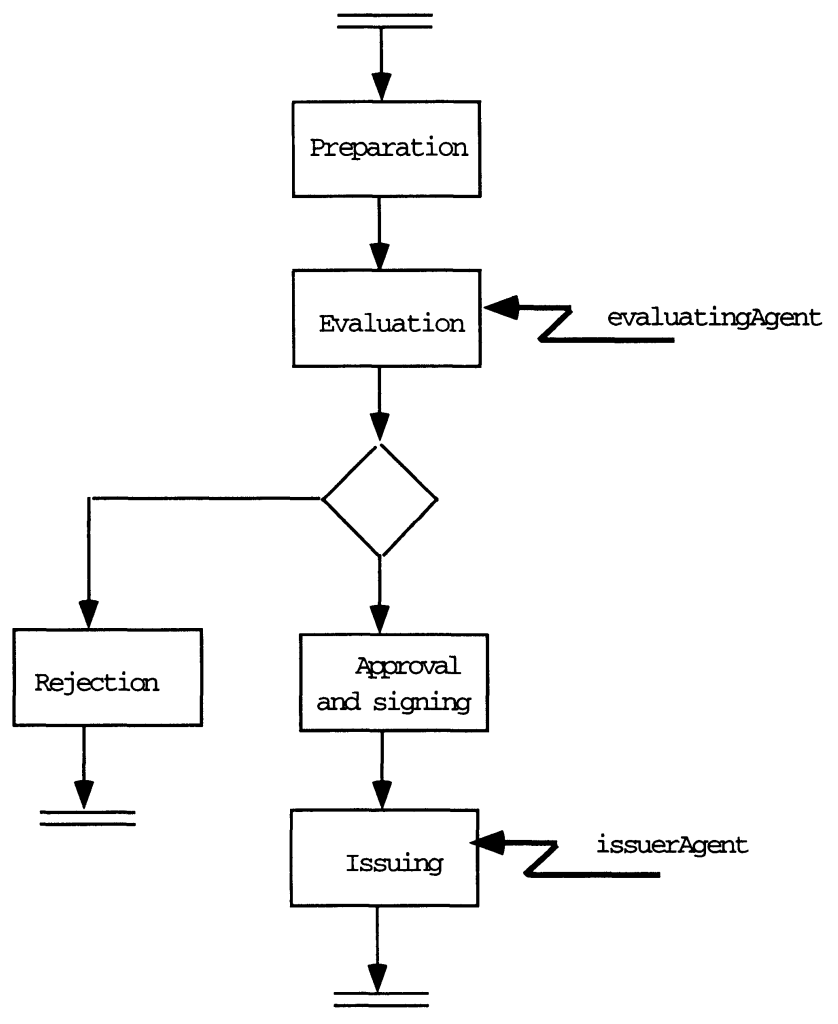

Figure 4.1 An example of Document Preparation workflow

on patterns and triggers are tackled. Finally, concluding remarks and future developments are given.

\subsection{USING RULES FOR EXCEPTION HANDLING IN WIDE}

In this section, we briefly review the basic concepts of the WIDE WF model; then, we concentrate on the structure of rules for treating exceptions, and on rules for specifying authorization constraints to cope with typical WF security requirements.

\subsubsection{Overview of the WIDE model}

In WIDE, a process is a WF schema defined as a collection of tasks which are the elementary work units. Tasks are organized into a flow structure defining the execution dependencies among tasks. The flow structure is specified by means of a restricted number of constructs allowing sequences, alternatives, and parallelism. Each WF schema has one start symbol and several stop symbols; 
the start symbol has one successor and each stop symbol has one predecessor. A WF schema may include the definition of structured data, represented by WF variables. WF variables, besides enabling information exchange among tasks of the same case, are accessed by the WF Management System (WFMS) for checking possible constraints and for determining the tasks to be scheduled, when conditional executions are specified. For a detailed description of WIDE constructs the reader can refer to [4]. A WF case is an execution of a WF schema, i.e., an instance of the corresponding WF schema. Multiple cases of the same process may be active at the same time. A case is executed by scheduling tasks (as defined by the flow structure) and by assigning them for execution to a human or an automated agent. As a case is started, the first task (the successor of the start symbol) is activated. As a task connected to a stop symbol is completed, the case is also completed.

An example of WF specification using the WIDE model is shown in Fig. 4.1. This refers to a simple Document Preparation WF, composed of five tasks: Preparation, Evaluation, Rejection, Approval and signing, and Issuing. As the WF starts, the Preparation task is executed under the constraint evaluatingAgent (depicted by an arrow that represents a WIDE trigger), and its completion will cause the starting of the Evaluation task. After this task ends, either the rejection procedure (after which the flow terminates) or the approval procedure can be executed but not both (conditional fork with mutual exclusion), depending on the outcome of the Evaluation task. An approved and signed document will then be issued to the final destination (Issuing task) under the issuerAgent constraint, and the flow terminates.

Rules and constraints, which are the main issue of this paper, will be explained in detailed in the following sections.

\subsubsection{Rules in WIDE}

The WIDE approach to WF design consists in modeling separately the normal behavior of a WF and the predictable deviations, or exceptions, of the normal behavior of the WF. Rules (or triggers) in WIDE are employed to specify and manage exceptions. Rules conforming to the ECA paradigm: the event part defines when the rule is triggered; the condition part verifies if the triggered rule needs to react to the triggering event, while the action part specifies the operations required to manage the event.

Rules in WIDE are specified in the object-oriented language Chimera-Exc [6]. Chimera-Exc requires that the object-oriented schema upon which rules execute is defined. Chimera-Exc rules exploits three types of classes: WIDE classes, WF-specific classes, and event handling classes.

WIDE classes include description of the organization (roles, agents, and so on), and description of tasks and cases. These classes are WF-independent, and are predefined in the system; objects are created when new roles, agents, tasks or cases are created. For instance, Chimera-Exc rules may refer to attributes of running cases by accessing the attributes of the WIDE class case (e.g., $\operatorname{case}(C)$, agent (A), C.responsible=A, A.name= "John' ' 
selects the case(s) whose responsible is John).

$W F$-specific classes store WF variables. Each case will be represented as an object within this class, created when the case is started.

Event handling classes store information carried by occurred events. For instance, the externalEvent class is referred to access the parameters of an occurred external event. A more detailed description of the WIDE specification language Chimera-Exc can be found in [6].

Event part. Each rule in Chimera-Exc can monitor multiple events, with a disjunctive semantics: the rule is triggered if any of its triggering events occurs. Events in Chimera-Exc, corresponding to the types of events previously listed, are specified as follows: i) data events, raising in correspondence of data manipulation primitives create, update, delete (e.g., constraint violation, task/case cancellation, unavailability of an agent); ii) external events, raised by external applications through the raise primitive (e.g., document arrival, telephone call, incoming e-mail); iii) WF events, enabling the monitoring of task/case starts and completions, expressed through the predefined events caseStart, caseEnd, taskStart (taskname), taskEnd(taskname); iv) temporal events, expressed as deadlines, time elapsed since a certain instant, or cyclic periods of time using the Chimera-Exc syntax.

Condition part. A condition is a predicate on the state of the WIDE database at the time of the condition evaluation which indicates whether the event must be managed. Rule condition includes class formulas (for declaring variables ranging over the current extent of a class, e.g., $\operatorname{tr}(\mathrm{C}) ; \mathrm{C}$ in this case ranges upon object identifiers of the tr class), type formulas (for introducing variables of a given type, e.g., integer(I)), and comparison formulas, which use binary comparison between expressions (e.g., T. executor= 'John' '). Terms in the expressions are attribute terms (e.g., C.destination) or constants. The predicate occurred, followed by an event specification, binds a variable defined on a given class to object identifiers of that class which were affected by the event. For instance, in agent (A), occurred(create(agent), A), A is bound to an object of the agent class that has been created. If the result of a query is empty (i.e., no bindings are produced), then the condition is not satisfied and the action part is not executed. Otherwise, bindings resulting from the formula evaluation are passed to the action part in order to perform the reaction over the appropriate objects.

Action part. The action (or reaction) part can contain notifications to one or more agents or corrective actions on the current execution, expressed through the following Chimera-Exc primitives: i) Chimera data-manipulation primitives: these allow the creation of an object via the create primitive, the modification of the value of an object's attribute via the modify primitive, or the deletion of an object via the delete primitive. For instance, delete (agent, A) removes all objects of class agent to which variable $\mathrm{A}$ is bound after the con- 


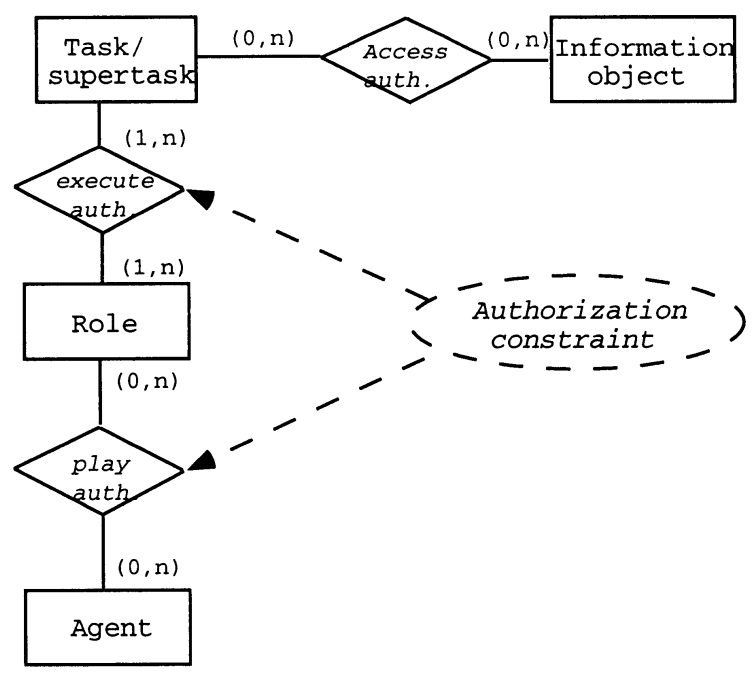

Figure 4.2 The organizational model of WIDE

dition evaluation. ii) Operation calls to the WF engine: these include primitives for notification of alarms to agents, starting a case or a task, and for assigning, re-assigning, rejecting, canceling, or rollbacking tasks or cases (e.g., notify (C.responsible, 'agent is unavailable' '), reassignTask(T)).

In the next section, we will describe the use of ECA rules to detect and manage exceptions violating authorization constraints on the execution of tasks by agents.

\subsection{AUTHORIZATION CONSTRAINTS AND RULES}

Assignment of tasks to agents is performed on the basis of the organizational model of WIDE which is shown in Fig. 4.2 using the Entity-Relationship notation. Only authorized agents can execute tasks. Moreover, the concept of role is introduced, according to the concepts defined in [12], to represent the capability of an agent to execute a task. According to this model, authorizations for agents to play roles and for roles to execute tasks are defined in the system, represented by the play authorization and execute authorization relationships, respectively. These authorizations are defined to reflect specific organization policies and rules, and task assignment is performed in respect of the defined authorizations. This way, the "need-to-know" and the "task confinement" principles can be enforced in the system [3]. According to the need-to-know principle, agents are constrained to execute only the task(s) of their competence, and each agent can access only the information necessary for the completion of the task(s) he/she is authorized for.

According to the task confinement principle, agents are constrained to access 


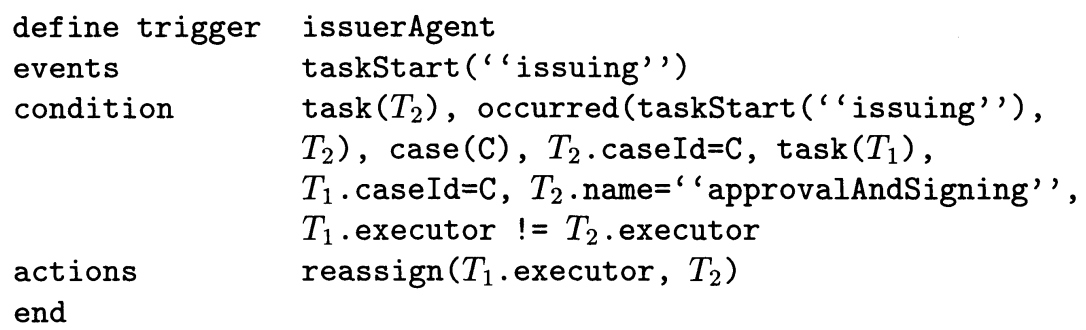

Figure 4.3 Specification of a trigger enforcing the binding of duties constraint

information objects only during the execution of a task. In fact, agents can access data objects only after their assignment to a given task, and any attempt to access data outside an authorized task is rejected. In addition to these two basic principles, authorization constraints can be imposed in the system, to enforce other security policies to flexibly regulate task assignment and execution, to cope with WF security requirements, in analogy with other approaches in the literature $[1,2,9]$. In particular, the following categories of constraints are considered:

- Constraints on agents, concerning the assignment of agents to roles for task execution. In particular, an authorization model for WF must support different types of constraints on agents. Examples of constraints on agents are: i) "Two different agents must execute two tasks $T_{1}$ and $T_{2}$ " to enforce a separation of duties constraint and ii) "The same agent must execute two tasks $T_{1}$ and $T_{2}$ " to keep the involved information confidential, realizing a "binding of duties" constraint.

- Constraints on roles, concerning the execution of tasks by roles. In particular, an authorization model for WF must support different constraints for task assignment and execution. An example of role constraint is the following: "At the least $K$ roles must be associated with the WF in order to start its execution", to enforce a "cooperation" constraint on a WF.

Authorization constraints on agents and roles are not statically defined in WIDE, since a specification language for this purpose is not available in the environment. Rather, in analogy with the general specification paradigm adopted in this system, we enforce authorization constraints by means of active rules. Active rules are defined to detect the exceptions representing possible violations to authorization constraints, and to properly react to detected exceptions.

For example, with reference to the WF of Fig. 4.1, a "binding of duties" constraint on the task Issuing imposing that the agent executing this task must be the same agent who executed the task Approval and sign, is enforced by means of the Chimera-Exc trigger issuerAgent whose specification code is shown in Fig. 4.3. The event part specifies that the trigger is raised as the Issuing task starts. The condition determines which is the instance involved 
by declaring two variables $T_{1}$ and $T_{2}$, both ranging over the tasks classes. The occurrence of the predicate restricts $T_{2}$ to range over the task for which the event taskStart has been raised. Further, it requires that the case of this task be the same as the one of $T_{1}$ (i.e., Approval and signing) and checks if their executor agents are different. In this case, the action associated with the trigger consists in reassigning to $T_{2}$ the agent of $T_{1}$.

Rules in WIDE can be associated with a given WF at different levels (e.g., task level, schema level), or independently of any WF, therefore affecting multiple schemas. For security, we focus on the first kind of rules, which are the most common ones in modeling authorization constraints. Task level rules capture exceptions related to a single task. For instance, an exception defining a warning to the security officer as a reaction to a task activation by an unauthorized agent should be declared associated with the task itself, as in the "binding of duties" trigger example.

Rules should instead be declared at the schema level if they enforce a security constraint affecting the entire WF. For instance, the constraint that $K$ roles must be associated with the WF should be enforced by means of a rule declared at the schema level, since a whole case is affected.

\subsection{AUTHORIZATION PATTERNS}

Defining security rules for all possible authorization constraints to be enforced in a WF can become cumbersome, specially when complex flows are specified, with several involved tasks and agents. On the other hand, typical authorization constraints that need to be enforced in a WF can be prefigured, as in the examples illustrated in the previous section. Therefore, the idea consists in identifying the skeleton of a rule enforcing a given authorization constraint and in properly packaging such skeleton into an authorization pattern. Authorization patterns predefine typical (sets of) rules capturing the knowledge about the exceptions (i.e., violations) to given authorization constraints and the actions that can be performed to react to them. Authorization patterns can then be used as the starting point for designing authorization rules in each situation where an authorization constraint applies.

Authorization patterns are defined according to a reference model composed of the following elements:

- The pattern specification, which is a description of the authorization constraint enforced by the pattern, and is composed of several parts. Some parts (i.e., name, intent, and classification) allow the designer to identify and understand the goal of the pattern. The template part allows the description of the pattern itself. Finally, the keywords, related to, and guidelines parts are defined to allow the designer to locate the pattern in a repository, to understand the links with other WF patterns available in WIDE, and to provide suggestions about possible usages and personalization of the pattern for trigger definition. The template part contains the core specification of the pattern, in terms of events, conditions, and ac- 


\section{Pattern Specification}

Name: bindingOfDuties

Intent: This pattern checks that agent executing task T2 is the same as the agent executing a previous task $T 1$ in a given WF, to enforces information confidentiality in the two tasks thorugh the binding of duties constraint.

Classification: Authorization patterns |Agent authorization patterns

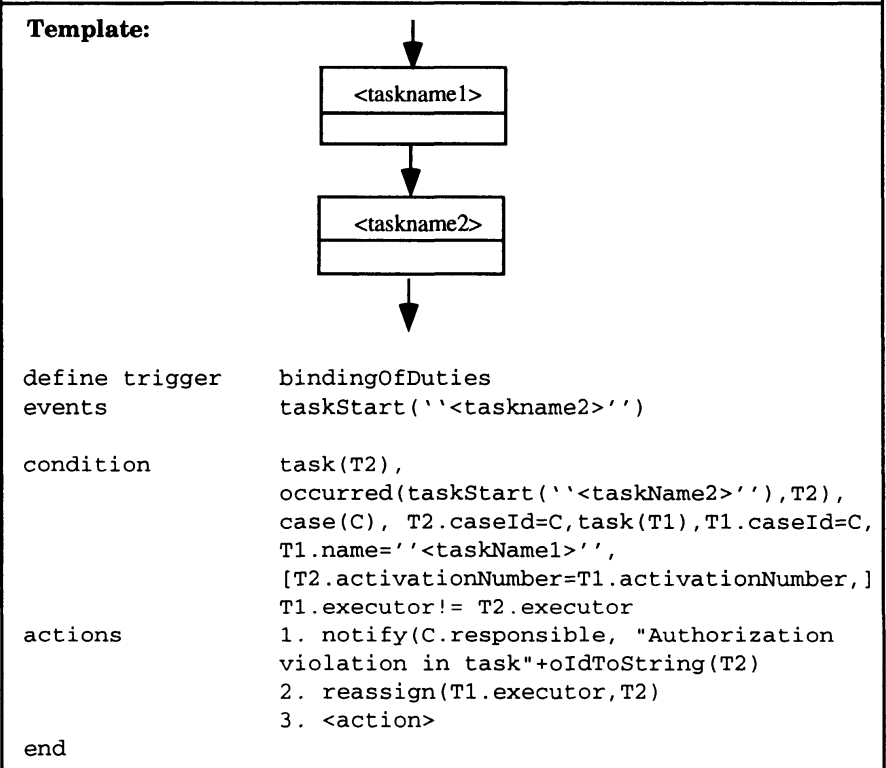

Keywords: security, separation of duties, violation, audit

Related to: integrity, roleExamination

Guidelines: The condition part dealing with activitation numbers is needed only if the trigger is attached to a piece of WF with a loop inside.

Figure 4.4 The binding0fDuties authorization pattern 
tions. The template contains parametric fields to be filled in with specific values provided by the designer. Mandatory and optional parts can also be specified in a template. Events and conditions represent the main elements of the pattern, since they describe how to capture exceptions in a generic way. The action element provides in general a list of suggestions. In fact, reactions to exceptions are in general application-dependent, and the most suitable action must be selected depending on the specific situation.

- Sample usages, which are pattern instantiations on specific application examples. These examples show how an authorization pattern can be personalized in different contexts and applications by illustrating how variables/parameters appearing in the "template" field of the pattern specification can be supplied by the designer to produce a concrete authorization trigger.

- Template interface, which is a user-oriented interface simplifying the pattern instantiation process by providing default values for variables/parameters. Its purpose is to hide syntactic details of the Chimera-Exc language while compiling a pattern within a given application.

To capture the constraints on agents and roles previously discussed, the following basic set of authorization patterns is provided in the WIDE catalog:

- bindingOfDuties pattern

- separation0fDuties pattern

- number0fRoles pattern

whose specifications are shown in Fig. 4.4, Fig. 4.5, and Fig. 4.6, respectively.

The separation0fDuties and the binding0fDuties patterns specify generic rules to enforce agent constraints, to guarantee that the executing agents of the two tasks are different or are the same, respectively. The number0fRoles pattern models the exceptions that can arise when violating the constraint on a minimum number $K$ of roles required to start the execution of a WF, which is an example of role constraint. It checks whether the number of roles appearing in the Roles attribute of the object task is not lower than a pre-defined minimum value stored in the minNumber0fRoles variable. When the pattern is used to generate a trigger, the value for this variable is set for each specific case to be checked.

In a pattern template, predefined parts, parameterized parts, and optional parts are defined. With reference to Fig. 4.4, we observe that the event and conditions clauses of the pattern template are parametric, that is, they are expressed in Chimera-Exc using generic parameters to become independent of any WF specific task. Generic parameters are specified within the " $<>$ " symbols. Moreover, an optional part (shown between the symbols [] in the figure) is specified in the condition clause of the pattern, related to the activation number, which should be used only if a rule has to be defined for WFs with a loop, as suggested by the Guidelines part of the specification. 


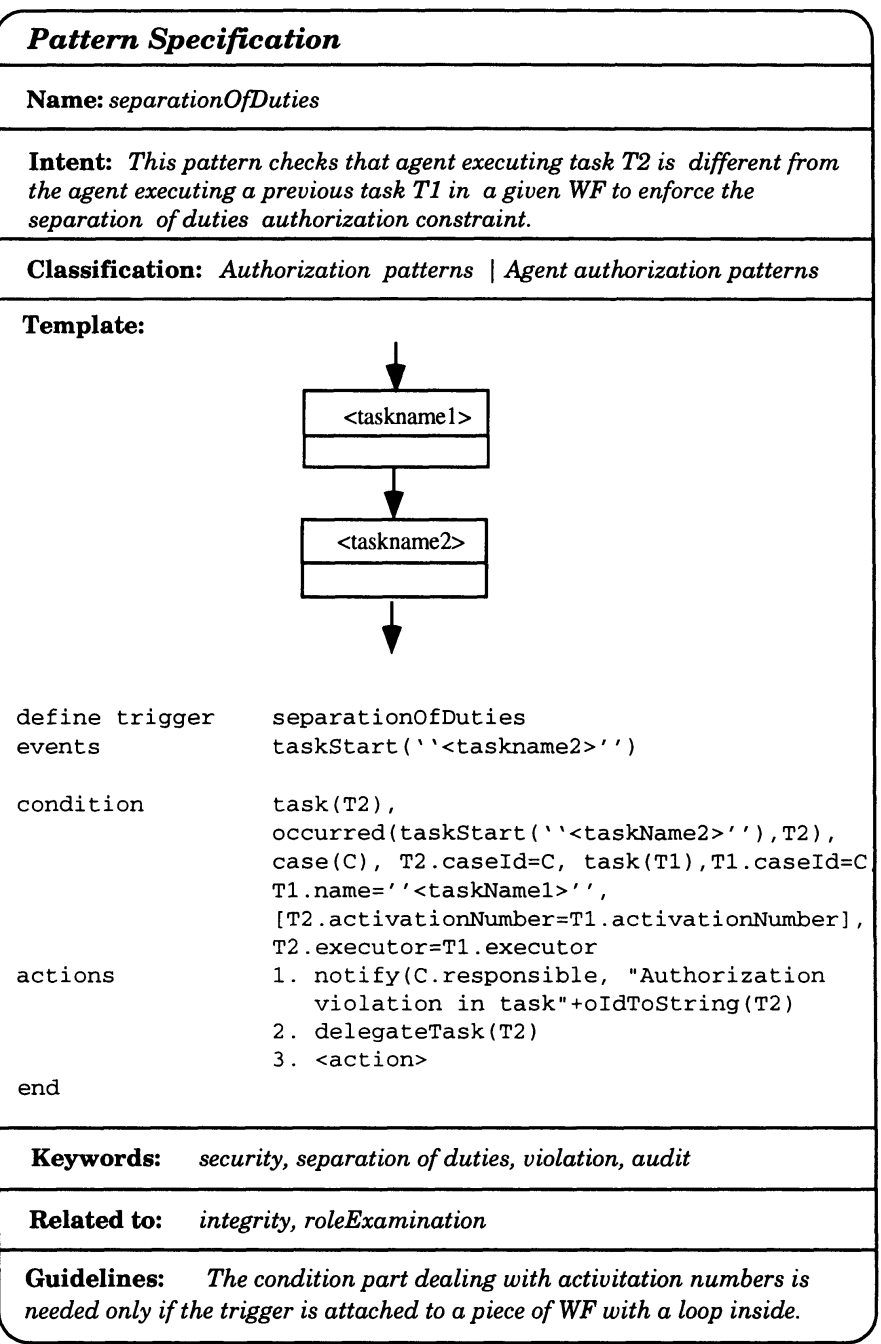

Figure 4.5 The separation0fDuties authorization pattern 


\section{Pattern Specification}

Name: numberOfRoles

Intent: This pattern counts the number of roles that are associated with a WF to allow WF starting only if a minimum number of roles is involved, to enforce a "cooperation" constraint.

Classification: Authorization patterns | Roles authorization patterns

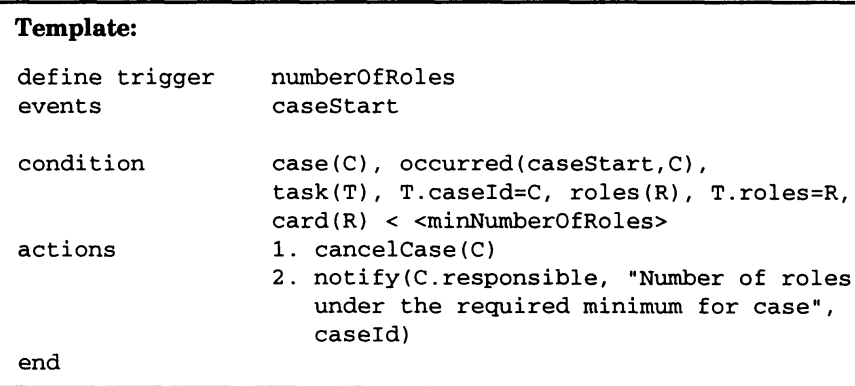

Keywords: security, number of roles, violation, audit

Related to: integrity, roleExamination

Guidelines: Besides case cancellation, also a notification message can be chosen for audit purposes.

Figure 4.6 The number 0 fRoles authorization pattern 


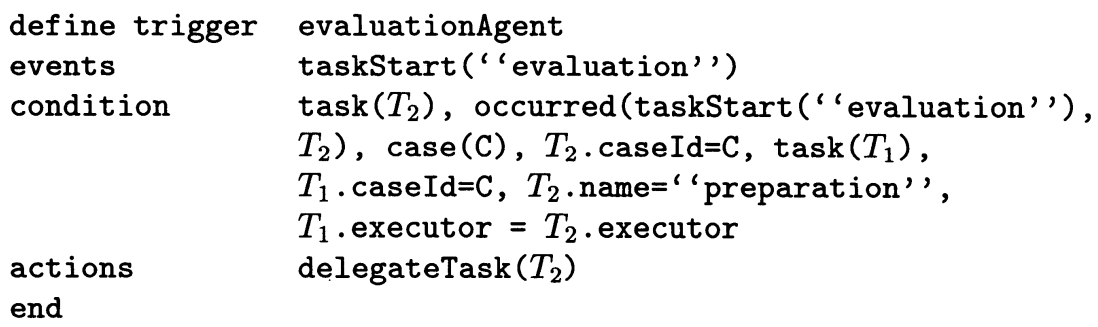

Figure 4.7 Example of pattern instantiation - the evaluationAgent trigger

Since a pattern provides a generalized description of the rule(s) necessary to enforce a given security constraint, it can be used for defining new rules of this kind in different WFs. The process by which a pattern is (re)used for generating new rules targeted to a specific WF is called pattern instantiation.

\subsubsection{Pattern instantiation}

Pattern instantiation is a mechanism for creating triggers to enforce a given authorization constraint on specific tasks and cases starting from an available pattern. Instantiation consists in binding all the parameterized parts of a pattern according to the desired usage of the trigger. The instantiation is based on a set of rules, that act on the event and condition parts of the pattern template, and on a set of constraints that must be verified to guarantee the correctness of the instantiation. A formal description of the instantiation rules and constraints is presented in [6]. Basically, such rules and constraints guarantee that all generic parameters that appear in the events and condition parts of a trigger in the pattern template are properly bound to corresponding Chimera-Exc expressions. An example of instantiation of the pattern binding0fDuties of Fig. 4.4 is the trigger issuerAgent shown in Fig. 4.3. In this example, parameters <taskname1> and <taskname2> have been instantiated into Issuing and Approval and signing, respectively, which are the tasks to which the binding of duties constraint must be applied. Moreover, only the reassignment action has been selected for these two tasks of the Document Preparation WF.

As another example of instantiation, the trigger of Fig. 4.7 is obtained from the separation0fDuties pattern to implement the evaluationAgent trigger associated with the task Evaluation of the WF of Fig. 4.1, requiring that the agent who evaluates a document be different from the one who prepared it.

A tool called WERDE (Workflow Exceptions Reuse and Design Environment) has been developed to support the management of the catalog of patterns in WIDE. The tool provides functionalities to access and manage the pattern catalog, that is, to retrieve patterns considered useful for a given application, to store new patterns (possibly with associated sample usages), and to remove existing patterns. The extensibility of the catalog is an important aspect, to allow the insertion of new patterns related to new authorization constraints of 
interest. In fact, there can be many different types of authorization constraints for which a suitable pattern has to be defined and made available in the catalog.

\subsection{ENFORCING AUTHORIZATION CONSTRAINTS THROUGH TRIGGERS}

In this section, we briefly discuss issues related to WF execution in presence of triggers enforcing authorization constraints. According to the constraint classification proposed in [2], three different types of constraints can be identified in a WF: i) static constraints, which can be evaluated before WF execution; ii) dynamic constraints, which can be evaluated only during WF execution, and iii) hybrid constraints, which can be partially evaluated without executing the WF. Constraints of type i) and iii) have the advantage of avoiding the execution of the WF if a violation occurs; they rely on the possibility of statically declaring constraints with some language. Since we adopt a pattern and trigger-based approach to constraint enforcement, we can not evaluate a constraint before the execution of a WF. However, we can associate the patterns related to static constraints with the caseStart event, which is the first event occurring in the system upon activation of a WF instance. Triggers are then evaluated as the caseStart event raises, avoiding the continuation of the whole case if a violation occurs. For example, the constraint that $K$ roles must be involved to start a WF is static, and, in fact, our numberOfRoles pattern is declared at the WF level, associated with the caseStart event, and avoids to start the execution of the case if this constraint is not met at the case starting.

Constraints on the agents executing two different tasks in sequence are dynamic, and the corresponding patterns are associated with the involved tasks and are evaluated as their execution starts.

As for hybrid constraints, they can be managed using two different patterns: a pattern associated with the caseStart event handling the exceptions related to the constraints that should be evaluated as soon as the case starts, and another pattern expressing the exceptions related to situations that can be checked only during case execution. An example of hybrid constraint is the one requiring that if at least $K$ roles are necessary to start the WF, and that tasks $T_{1}$ and $T_{2}$ must be executed by two different agents. This constraint can be enforced using the numberOfRoles pattern first, to check the first part of the constraint. Then, if this constraint is satisfied (i.e., no exceptions occurred), the separationofDuties pattern can be used to define a trigger for controlling the second part of the constraint during flow execution.

\subsection{CONCLUDING REMARKS}

In this paper, we have presented an approach to design authorization constraints in WF systems based on the use of rules to be executed by the WF active database. Moreover, we have shown how these rules can be constructed for a WF by instantiating predefined rule skeletons called authorization patterns. Authorization patterns describe the knowledge about violations detection and 
handling in a general way, to bounded to appropriate values in order to be included in a WF as triggers. A tool called WERDE has been implemented to support pattern definition and usage in WIDE; the tool operates on a pattern catalog, where authorization patterns are properly stored and classified. The tool exploits the pattern interface to guide the designer in completing a correct and executable WF, including the verification of authorization constraints which become WF triggers.

Currently, we are working on the extension of authorization patterns in the catalog, with, for example, patterns enforcing the separation and binding of duties constraints on roles, to extend the situations that can be controlled and make the system more flexible. We are also studying problems related to access control to information objects in external information systems interfaced by the WF. We are implementing a set of functionalities for schema analysis in presence of authorization triggers and other security functionalities for WFs, such as private and public key encryption algorithms for WF data confidentiality and for user certification. For this purpose, the tool will be linked to the functionalities of another tool which has been implemented for security management in the context of the CNR DEMOSTENE Project devoted to security of distributed systems in the Public Administration domain.

\section{Acknowledgments}

This work has been partially supported by the Italian Consortium for Informatics (CINI) and by the Italian National Research Council in the framework of the "DEMOSTENE Project". We thank Dr. Eng. Fabio Casati who collaborated in revising security rules and Dr. Angelica Tescaro who collaborated to part of this work during her degree thesis.

\section{References}

[1] V. Atluri, W.K. Huang, "An Authorization Model for Workflows", in Proc. of ESORICS'96, 4th European Symposium On Research in Computer Security, Rome, Italy, September 1996.

[2] E. Bertino, E. Ferrari, V. Atluri, "A Flexible Model Supporting the Specification and Enforcement of Role-based Authorizations in Workflow Management Systems", in Proc. of 2nd ACM Workshop on Role-Based Access Control, November 1997.

[3] S. Castano, M.G. Fugini, G. Martella, P. Samarati, Database Security, Addison Wesley, ACM Press, 1995.

[4] S. Castano, M.G. Fugini, I. Mirbel-Sanchez, B. Pernici, "Workflow Reference Models", WIDE Deliverable n. 5.2.2, 3015-1, May 1997.

[5] S. Castano, S. De Capitani Di Vimercati, M.G. Fugini, "Automated Derivation of Global Authorizations for Database Federations", Journal of Computer Security, Vol.5, No.4, 1997, IOS Press. 
[6] F. Casati, S. Castano, M.G. Fugini, I. Mirbel-Sanchez, B. Pernici, "Using Patterns to Design Rules in Workflows", Internal Report n. 97-065, Dip. Elettronica e Informazione, Politecnico di Milano, 1997 (submitted for publication).

[7] E. Gamma, R. Helm, R. Johnson, J. Vlissides, Design Patterns - Elements of Reusable Object-Oriented Software, Addison Wesley, 1994.

[8] D. Georgakopoulos, M. Hornik, A. Sheth, "An Overview of Workflow Management: From Process Modeling to Workflow Automation Infrastructure", Distributed and Parallel Databases,, Kluwer Academic Publishers, Vol.3, 1995.

[9] E. Ellmer, G. Pernul, G. Quirchmayr, "Security for Workflow Management", in Proc. of 6th IASTED/ISMM Int. Conf. on Parallel and Distributed Computing and Systems, Washington D.C., October 1994.

[10] R.S. Sandhu, "Separation of Duties in Computerized Information Systems", in Database Security IV: Status and Prospects, S. Jajodia and C. Landwehr (Eds.), North-Holland, 1991.

[11] R.S. Sandhu, "Task-Based Authorization: A Paradigm for Flexible and Adaptable Access Control in Distributed Applications", in Proc. of 16th NIST-NCSC National Computer Security Conference, Baltimore, MD, September 1993.

[12] R.S. Sandhu, E.J. Coyne, H.L.Feinstein, C.E.Youman, "Role-Based Access Control Models", IEEE Computer, February 1996.

[13] J. Widom, S. Ceri, Active Database Systems: Triggers and Rules for Advanced Applications, Morgan and Kaufmann, San Mateo, CA, 1996. 